\title{
Extension of the generalized multipole technique to anisotropic medias
}

\author{
Nicolas B. Piller, Olivier J.F. Martin \\ Laboratory for Field Theory and Microwave Electronics, Swiss Federal Institute of Technology, ETH-Zentrum, 8092 Zurich, Switzerland
}

Received 22 September 1997; revised 26 November 1997; accepted 27 November 1997

\begin{abstract}
This paper presents an extension of the generalized multipole technique (GMT) for 2D anisotropic scatterers. New expansions similar to the Bessel multipole expansion are derived for arbitrary anisotropic media. Numerical simulations prove the accuracy and the rapid convergence of these expansions. As the results obtained are extremely accurate, this technique is most helpful for the evaluation of reference solutions and for the understanding of the physical interaction of light with arbitrary anisotropic media. (C) 1998 Elsevier Science B.V. All rights reserved.
\end{abstract}

\section{Introduction}

The generalized multipole technique (GMT) is a powerful method for 2D and 3D scattering calculations [1-5]. It has been used for the study of numerous physical problems, such as for example antennas [6], near field microscopy [7], waveguides [8], scattering by particles [9], and absorption by human bodies $[10,11]$.

Over the last few years this technique was extended to handle different situations, as periodic scatterers [12], static problems [13] or the scattering of a Gaussian beam [14]. In this Letter, 2D anisotropic expansions are derived for GMT. As they include arbitrary anisotropy, magneto-optic scatterers can also be considered.

The theory of GMT is summarized in Section 2. The expansions for anisotropic media are derived in Section 3. Numerical simulations including comparisons with coupled-dipole approximation (CDA) results are presented in Section 4. Finally the results are summarized in Section 5.

\section{The generalized multipole technique}

Let us consider a scattering system embedded into an infinite homogeneous system $\mathscr{D}_{0}$ and illuminated by an incident field $\psi^{0}(\boldsymbol{r})=\left\{\boldsymbol{E}^{0}(\boldsymbol{r}) ; \boldsymbol{H}^{0}(\boldsymbol{r})\right\}$. The scatterer is decomposed into a series of homogeneous domains $\mathscr{D}_{i}$.
For each domain the total field $\psi^{\mathscr{D}_{i}}(\boldsymbol{r})=\left\{\boldsymbol{E}^{\mathscr{D}_{i}}(\boldsymbol{r}) ; \boldsymbol{H}^{\mathscr{D}_{i}}(\boldsymbol{r})\right\}$ is represented by an expansion formed by a set of $N^{\mathscr{D}_{i}}$ functions $\psi_{n}^{\mathscr{D}_{i}}(\boldsymbol{r})$ :

$\psi^{\mathscr{D}_{i}}(\boldsymbol{r})=\sum_{n=1}^{N^{\mathscr{D}_{i}}} a_{n}^{\mathscr{D}_{i}} \psi_{n}^{\mathscr{D}_{i}}(\boldsymbol{r})$,

where $a_{n}^{\mathscr{D}_{i}}$ are unknown parameters and each function $\psi_{n}^{\mathscr{D}_{i}}(\boldsymbol{r})$ fulfills Maxwell equations in the corresponding domain. For the embedding medium $\mathscr{D}_{0}$ each function $\psi_{n}^{\mathscr{D}_{0}}(\boldsymbol{r})$ has also to fulfill the radiation conditions at infinity; furthermore, the incident field $\psi^{0}(\boldsymbol{r})$ is also taken into account in $\mathscr{D}_{0}$ :

$\psi^{\mathscr{D}_{0}}(\boldsymbol{r})=\psi^{0}(\boldsymbol{r})+\sum_{n=1}^{N^{\mathscr{D}_{0}}} a_{n}^{\mathscr{D}_{0}} \psi_{n}^{\mathscr{D}_{0}}(\boldsymbol{r})$.

The unknown parameters $a_{n}^{\mathscr{D}_{i}}$ are evaluated using the boundary conditions. More precisely, the boundaries between the different domains $\mathscr{D}_{i}$ are discretized and the parameters $a_{n}^{\mathscr{D}_{i}}$ are chosen such that the sum, over the discretization points, of the mismatch on the boundary conditions is minimized. This results in a linear system of equations to be solved with a least square algorithm. The system of equations has $N$ unknowns and $C D$ equations, where $N$ is the total number of parameters, $D$ the number of discretization points and $C$ the number of field components considered in the error definition ( 6 in a general $3 \mathrm{D}$ 
problem). To obtain secure results the system of equations has to be strongly over-determined.

The choice for the origin and order of the expansions is the main difficulty of GMT. Indeed the expansions must be able to represent the field inside the corresponding domain, otherwise the solution cannot converge. The convergence can be tested by observing the residual error on the boundary conditions. If this error is not small enough another simulation has to be performed with a better expansion set.

For a more detailed descriptions of GMT the interested reader is referred to Refs. [12] and [15].

\section{Anisotropic 2D expansions}

The most commonly used expansions for GMT are the multipoles [2]. While different kinds of multipole expansions exist, we restrict our study to the multipole expansion with Bessel function as radial dependence. This expansion is called Bessel multipole expansion (BME) and is generally well suited for any simply connected closed domain.

To derive the anisotropic 2D BME, the isotropic Bessel multipole is first represented by a plane wave expansion. This representation is then generalized for the anisotropic case, forming the expansion functions of the anisotropic 2D BME. Considering a special case of this representation, a second expansion is then derived to reduce the numerical requirements.

Considering the $\hat{z}$-direction as the translation symmetry of the $2 \mathrm{D}$ problem, the electric field of the $2 \mathrm{D}$ transverse magnetic (TM) Bessel multipole of order $m$ is [16]:

$\boldsymbol{E}_{m}^{\mathrm{TM}}(\boldsymbol{r})=k J_{m}(k|\boldsymbol{r}|) \mathrm{e}^{i m \phi} \hat{z}$,

where $\phi$ is the argument of the position vector $\boldsymbol{r}=(x, y)$, $k$ the wave number of the corresponding domain and $\hat{z}$ the unit vector pointing in the $\hat{z}$-direction. The TM-BME of order $M, \boldsymbol{E}^{\mathrm{TM}}(\boldsymbol{r})$, is the series of the function (3) with $m=-M, \ldots, M$ :

$\boldsymbol{E}^{\mathrm{TM}}(\boldsymbol{r})=\sum_{m=-M}^{M} a_{m} \boldsymbol{E}_{m}^{\mathrm{TM}}(\boldsymbol{r})$,

where $a_{m}$ are the expansion parameters.

Using the integral representation of the Bessel function [17], the Bessel multipole (3) becomes

$\boldsymbol{E}_{m}^{\mathrm{TM}}(\boldsymbol{r})=\frac{i^{-m}}{2 \pi} \int_{0}^{2 \pi} \boldsymbol{e}_{\theta}^{\mathrm{TM}}(\boldsymbol{r}) \mathrm{e}^{i m \theta} \mathrm{d} \theta$

where $\boldsymbol{e}_{\theta}^{\mathrm{TM}}(\boldsymbol{r})$ represents the electric field of a TM plane wave propagating in the $\theta$-direction:

$\boldsymbol{e}_{\theta}^{\mathrm{TM}}(\boldsymbol{r})=k \mathrm{e}^{i k(x \cos (\theta)+y \sin (\theta))} \hat{z}$.
Eq. (5) is the plane wave expansion of the Bessel multipole for isotropic media. For the anisotropic case, the isotropic plane waves in Eq. (5) are replaced by plane waves propagating in the corresponding anisotropic medium. Such a plane wave is given for example by Chew [18]. In an anisotropic medium, the wave number $k_{\theta}^{\mathrm{TM}}$ is a solution of an eigenvalue problem and the direction of the electric field $\boldsymbol{v}_{\theta}^{\mathrm{TM}}$ the corresponding eigenvector (actually a complementary solution $\left(k_{\theta}^{\mathrm{TE}}, \boldsymbol{v}_{\theta}^{\mathrm{TE}}\right)$ exists for the transverse electric (TE) polarization). The TM plane wave is then:

$\boldsymbol{e}_{\theta}^{\mathrm{TM}}(\boldsymbol{r})=k_{\theta}^{\mathrm{TM}} \mathrm{e}^{i k_{\theta}^{\mathrm{TM}}(x \cos (\theta)+y \sin (\theta))} \boldsymbol{v}_{\theta}^{\mathrm{TM}}$.

As an example, the solution $\left(k_{\theta}^{\mathrm{TM}}, \boldsymbol{v}_{\theta}^{\mathrm{TM}}\right)$ for an anisotropic medium with diagonal $\boldsymbol{\mu}$ and $\boldsymbol{\epsilon}$ tensors is

$k_{\theta}^{\mathrm{TM}}=\frac{\omega}{\sqrt{\mu_{x x}^{-1} \epsilon_{z z}^{-1} \sin ^{2}(\theta)+\mu_{y y}^{-1} \epsilon_{z z}^{-1} \cos ^{2}(\theta)}}$,

and

$\boldsymbol{v}_{\theta}^{\mathrm{TM}}=\hat{z}$.

As we see, it is unfortunately not possible to evaluate analytically the integral in Eq. (5) for the anisotropic plane wave (Eq. (7)). Therefore a numerical approximation for the anisotropic Bessel multipole has to be made, replacing the integral in Eq. (5) with a sum,

$\boldsymbol{E}_{m}^{\mathrm{TM}}(\boldsymbol{r})=\frac{i^{-m}}{P} \sum_{p=0}^{P-1} \boldsymbol{e}_{\theta}^{\mathrm{TM}}(\boldsymbol{r}) \mathrm{e}^{i m \theta}, \quad$ with $\theta=\frac{2 \pi p}{P}$,

where $P$ is the number of plane waves used for the approximation.

The computation of BME (Eq. (4)) requires a series of $\boldsymbol{E}_{m}^{\mathrm{TM}}(\boldsymbol{r})$ for $m=-M, \ldots, M$. As Eq. (10) represents a discrete Fourier transform, the evaluation of the series $\boldsymbol{E}_{m}^{\mathrm{TM}}(\boldsymbol{r})$ for $m=-M, \ldots, M$ can be strongly speeded up by using the fast Fourier transform (FFT). Choosing $P$ large enough, Eq. (10) gives an accurate representation of the anisotropic Bessel multipole in the considered domain and can therefore directly be used to build up the anisotropic BME (Eq. (4)). This anisotropic BME is tested in Section 4 with $P=16 \mathrm{M}$.

Actually, a large $P$ value is not mandatory. Indeed, as each anisotropic plane wave (6) fulfills the Maxwell equations, so does the approximation of the Bessel multipole (10). Therefore it can be used directly as GMT expansion without any loss of accuracy, even if $P$ is small. Yet, a lower limit for $P$ exists. Indeed, introducing Eq. (10) into Eq. (4) gives

$\boldsymbol{E}^{\mathrm{TM}}(\boldsymbol{r})=\sum_{p=0}^{P-1} b_{p} \boldsymbol{e}_{\theta}^{\mathrm{TM}}(\boldsymbol{r}), \quad$ with $\theta=\frac{2 \pi p}{P}$, 
where $b_{p}$ represents the weight of the $p$ th plane wave:

$$
\begin{aligned}
& b_{p}=\sum_{m=-M}^{M} a_{m} \frac{i^{-m}}{P} \exp \left(i m \frac{2 \pi p}{P}\right), \\
& \text { with } p=0, \ldots, P-1 .
\end{aligned}
$$

If $P<2 M+1$, Eq. (12) represents an underdetermined system of equations. This means that different values for the parameters $a_{m}$ can be chosen to represent the same weights $b_{p}$ and therefore the same field. Such underdetermined expansion is not suitable for GMT. Therefore the number of plane waves must at least be equal to the number of expansion functions (i.e. $P \geq 2 M+1$ ).

Considering the case where $P=2 M+1$, we remark that Eq. (12) is a fully determined system of equations. The parameters $a_{m}$ being determined by the parameters $b_{p}$ and vice-versa. This means that the expansion in Eq. (11) with the unknown parameters $b_{p}$, is totally equivalent to the approximation of the Bessel multipole expansion with $P=2 M+1$. We call this new expansion (11) plane wave expansion (PWE). The anisotropic plane waves are directly used in the PWE. This is advantageous from a numerical point of view as no summation or FFT is required. Numer-
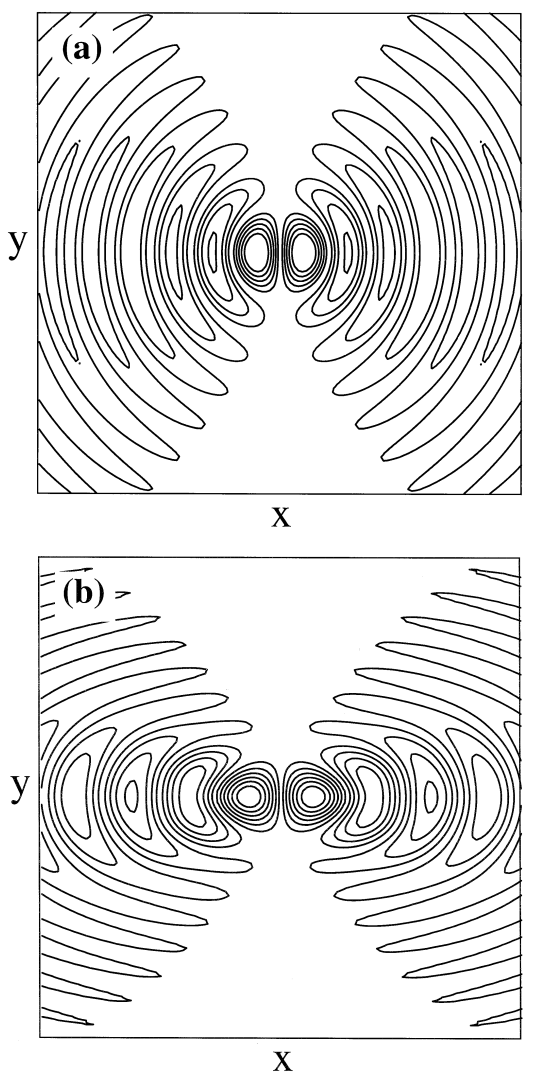

Fig. 1. TE Bessel multipole of order 1 for (a) isotropic, (b) anisotropic media. For the anisotropic medium $\epsilon_{x x}$ is four times $\epsilon_{y y}$ and all the non-diagonal elements vanish. ical simulations in Section 4 demonstrate the good accuracy with the PWE.

The magnetic field of the expansions is also required by the GMT. This field is simply obtained by using the magnetic field of the corresponding anisotropic plane waves [18].

Only the TM case has been explicitly derived in this section. The TE expansion is obtained in a similar way by considering the magnetic field instead of the electric field.

As illustration, the electric field of the TE Bessel multipole of order $m=1$ is represented in Fig. 1(a) for an isotropic medium and in Fig. 1(b) for an anisotropic medium. While the isotropic case shows circular symmetry (Fig. 1(a)), the anisotropic one has an elliptical structure corresponding to the ellipticity of the optical tensor (Fig. 1(b)).

\section{Simulations}

To assess the accuracy of the anisotropic BME and PWE, we compare numerical results with a reference solution obtained with the coupled-dipole approximation (CDA), a numerical method also known as discrete-dipole approximation (DDA) [19-21]. The physical problem is an anisotropic dielectric cylinder in vacuum, illuminated by a TM plane wave. The radius of the cylinder is equal to the wavelength of the embedding medium and the dielectric tensor of the cylinder shows strong non-diagonal elements:

$\boldsymbol{\epsilon}=\left[\begin{array}{rrr}2 & -i & 0 \\ i & 2 & 0 \\ 0 & 0 & 1\end{array}\right]$

Such a material is similar to magneto-optic materials [2224]. Strong non-diagonal elements have been expressly chosen to emphasize the anisotropic behavior while moderate diagonal elements ensure a very accurate reference solution by CDA.

Let us define the relative error

$\Psi(\boldsymbol{r})=\frac{\left\|\boldsymbol{E}(\boldsymbol{r})-\boldsymbol{E}^{\mathrm{ref}}(\boldsymbol{r})\right\|^{2}}{\left\|\boldsymbol{E}^{\mathrm{ref}}(\boldsymbol{r})\right\|^{2}}$,

between the electric field $\boldsymbol{E}(\boldsymbol{r})$ obtained by GMT and the electric field $\boldsymbol{E}^{\mathrm{ref}}(\boldsymbol{r})$ of the reference solution. In order to measure the overall accuracy of the computation we also define the global error $\langle\Psi\rangle$ as average value of $\Psi(\boldsymbol{r})$ over the entire scatterer.

The global error $\langle\Psi\rangle$ is reported in Fig. 2 as a function of the number of parameters $N^{\mathscr{D}_{\mathrm{c}}}$ for the expansion representing the field inside the cylinder, both for $\operatorname{BME}(M=$ $\left.\left(N^{\mathscr{D}_{\mathrm{c}}}-1\right) / 2\right)$ and PWE $\left(P=N^{\mathscr{D}_{\mathrm{c}}}\right)$. We first remark that, in both cases, the error is large for $N^{\mathscr{D}_{\mathrm{c}}}$ smaller than 15. This is caused by an expansion order too low to represent the field inside the cylinder. The results are inaccurate but this inaccuracy is pointed out by the GMT, the mismatch 


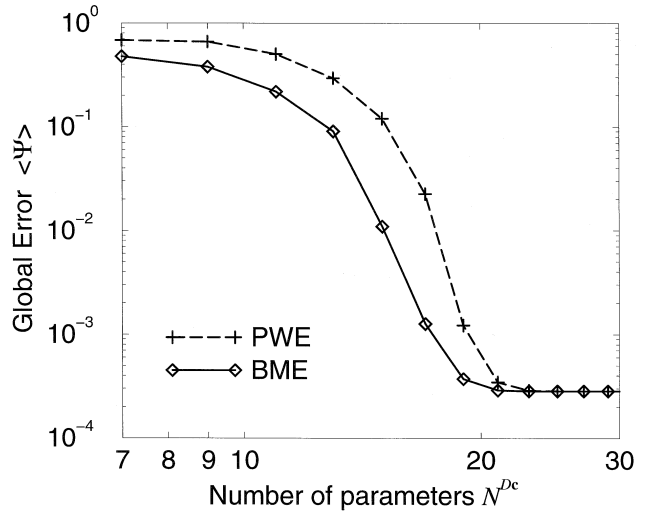

Fig. 2. Global error $\langle\Psi\rangle$ between the electric field computed with GMT and with CDA (reference solution), as a function of the number of parameters for the GMT expansion representing the field inside the scatterer. Both plane wave expansion (PWE) and Bessel multipole expansion (BME) are investigated. The scatterer is an anisotropic dielectric cylinder ( $\epsilon$ is given in Eq. (13)) of radius equal to the wavelength in the embedding media (vacuum) and is illuminated by a TE plane wave.

on the boundary conditions being large (Section 2). For $N^{\mathscr{D}_{\mathrm{c}}}$ larger than 15 the error $\langle\Psi\rangle$ decreases and converges rapidly. In this decreasing part, the horizontal distance between both curves is approximatively equal to 2 . This means that only 2 additional parameters are required by PWE to get the same accuracy as BME. For $N^{\mathscr{C}_{\mathrm{c}}}$ larger than 20 , a saturation of $\langle\Psi\rangle$ is visible (Fig. 2). This is caused by the finite precision of the reference solution. Although a very fine discretization was used for CDA ( 80 cells per wavelength in vacuum) the accuracy of GMT solutions becomes rapidly higher than the reference solution. For $N^{\mathscr{O}_{c}}>20$, the value of $\langle\Psi\rangle$ represents then the accuracy of the reference solution and the accuracy of GMT simulations (both for BME and PWE) are assumed to continue their convergence, as we have observed in the isotropic case using the MIE reference solution.

To prove that the reference solution is the real cause of the saturation of $\langle\Psi\rangle$, we effectuated the same convergence study as in Fig. 2 with different discretizations for the CDA reference solution. Using a 2 times finer, respectively rougher discretization we obtained exactly the same curves as in Fig. 2 except that the saturation of the convergence was 2 times lower, respectively higher. Furthermore, in Fig. 3(a) we observe for $N^{\mathscr{X}_{c}}=33$ that the relative error $\Psi(r)$ is concentrated on the object boundary. Such an error distribution is typical for CDA, as has been shown recently [25]. On the other hand, a GMT calculation with fewer parameters (e.g. $N^{\mathscr{O}_{\mathfrak{c}}}=17$ ), which corresponds to a less accurate representation of the field, does not produce an error concentrated on the object boundary but spread over the entire cylinder (Fig. 3(b)).

The convergence has been studied for different materials and types of anisotropy. In each case, the number of parameters required either by PWE or BME to obtain a given accuracy is very similar.

The electric field at the time $t=0$ computed by PWE with $N^{\mathscr{D}_{\mathrm{c}}}=33$ is represented in Figs. 4(b) and 4(c) and is compared to an isotropic example with $\epsilon=2$ (Fig. 4(a)). The incident field is a plane wave of unity amplitude propagating in the $\hat{x}$-direction for Figs. 4(a) and 4(b) and $-\hat{x}$ direction for Fig. 4(c). As the dielectric tensor is similar to that of magneto-optic media, interesting behaviors are observed in this figure. First we notice that the symmetry of the field is spoiled by the non-symmetry of the dielectric tensor (Figs. 4(b) and 4(c)). Second, observing the right-hand side of Fig. 4(b), respectively the lefthand side of Fig. 4(c) we notice that the field focus does not lie on the $\hat{x}$-axis as it was the case for the isotropic scatterer (Fig. 4(a)), but is shifted lower, respectively higher. This effect evidences a lack of symmetry representing a gyrotropic effect produced by the non-symmetric tensor. Third, observing in the left part of the cylinder the behavior of the field first for the isotropic case (Fig. 4(a)), we remark that the field simply oscillates, staying in the
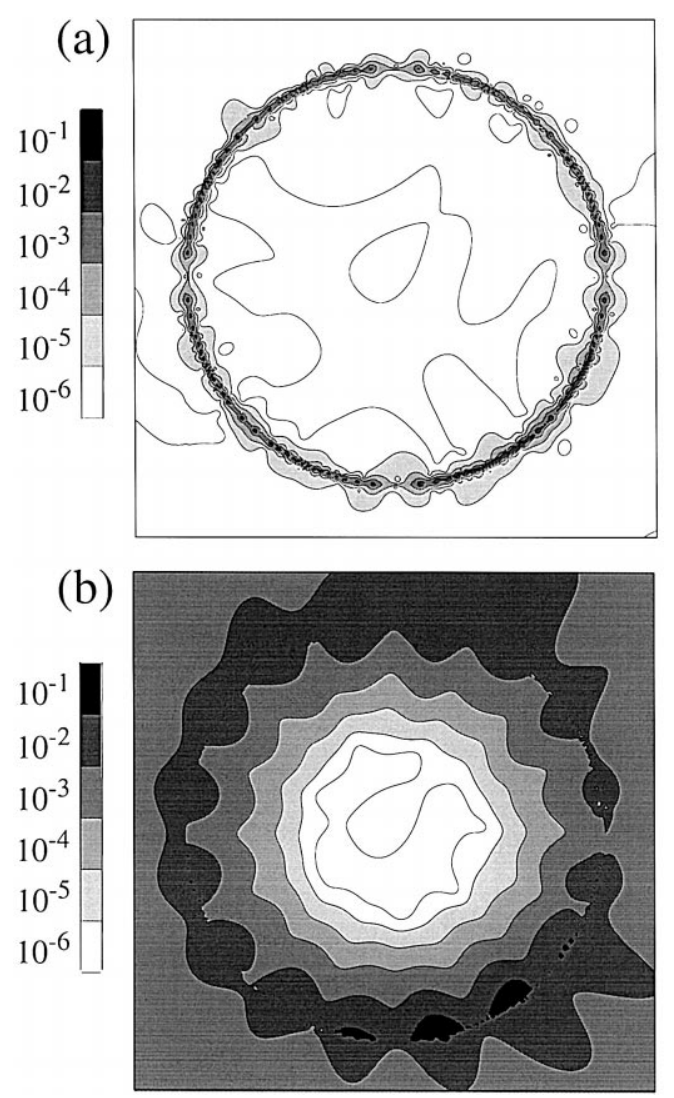

Fig. 3. Relative error $\Psi(\boldsymbol{r})$ between GMT and CDA computations of the physical problem described in Fig. 2. The field inside the anisotropic scatterer is represented by PWE with: (a) 33; (b) 17 parameters. 

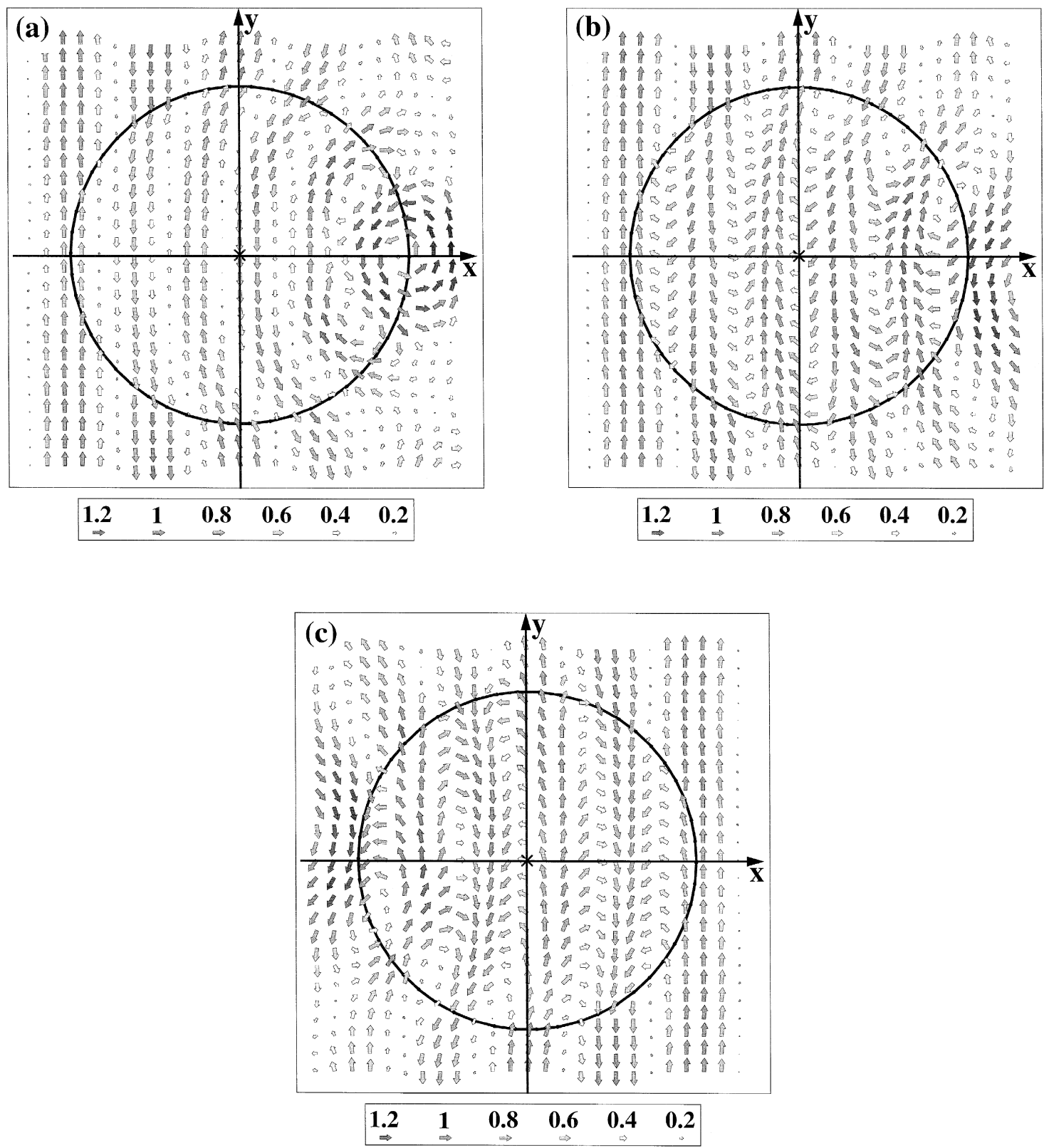

Fig. 4. Comparison of the electric field for (a) isotropic, (b), (c) anisotropic scattering problems. The incident field is a plane wave of unity amplitude propagating in the $\hat{x}$-direction (a), (b) and $-\hat{x}$-direction (c). The anisotropic problem is described in Fig. 2 and the isotropic one is identical except for the dielectric constant $\epsilon=2$. The field inside the scatterer is computed using PWE (33 parameters).

same direction and passing through a vanishing value, between two opposite maxima. A totally different behavior is visible for the anisotropic case (Fig. 4(b)), where the field rotates between the two maxima and never vanishes. Like this, if one observes the time behavior of the field at a fixed position, it simply oscillates for the isotropic case (Fig. 4(a)) while it rotates anticlockwise for the anisotropic one (Fig. 4(b)). The displacement field $\boldsymbol{D}(\boldsymbol{r})=\boldsymbol{\epsilon} \boldsymbol{E}(\boldsymbol{r})$ is also rotating but clockwise because the dielectric tensor in our example is antisymmetric [26].

\section{Conclusion}

New 2D expansions for anisotropic media have been derived for GMT. Based on a representation of the Bessel 
multipole by a finite number of plane waves, these expansions are well suited for any kind of anisotropy as long as the domain is closed. Efficient numerical implementations have been proposed. These expansions have been tested and have shown very fast convergence.

Because of the very good convergence of GMT, it is very well suited for the calculation of reference solutions. Like this, the new expansions presented in this paper should be helpful for the understanding of the physical interaction of light with arbitrary anisotropic media.

\section{Acknowledgements}

It is a pleasure to acknowledge fruitful discussions with R. Ballisti, C. Hafner, P. Leuchtmann and R. Vahldieck. This work was supported by the Swiss National Science Foundation (NFP 36).

\section{References}

[1] C. Hafner, The Generalized Multipole Technique for Computational Electromagnetic, Artech House, Boston, 1990.

[2] C. Hafner, L. Bomholt, The 3D Electromagnetic Wave Simulator, Wiley, Chichester, 1993.

[3] Yu.A. Eremin, N.V. Orlov, A.G. Sveshnikov, Sov. Phys. Dokl. 37 (1992) 31.

[4] Yu.A. Eremin, A.G. Sveshnikov, Electromagnetics 13 (1993) 1.

[5] Y. Leviatan, Z. Baharav, E. Heyman, IEEE Trans. Antennas Propag. 43 (1995) 1091.

[6] R. Yew-Siow Tay, N. Kuster, ACES J. 9 (1994) 79.
[7] L. Novotny, D.W. Pohl, B. Hecht, Optics Lett. 20 (1995) 970.

[8] C. Hafner, Int. J. Numer. Model. El. 3 (1990) 247.

[9] Yu.A. Eremin, N.V. Orlov, V.I. Rozenberg, Opt. Spectrosc. 73 (1992) 573.

[10] N. Kuster, ACES J. 7 (1992) 43.

[11] N. Kuster, IEEE Trans. Bio-Med. Eng. 40 (1993) 611.

[12] C. Hafner, J. Opt. Soc. Am. A 12 (1995) 1057.

[13] M. Gnos, P. Leuchtmann, A new MMP-code for static field computation, in: 11th Annual Review of Progress in Applied Computational Electromagnetics (ACES), Monterey, CA, March 1996, vol. 1, pp. 81-88.

[14] T. Evers, H. Dahl, T. Wriedt, Electron. Lett. 32 (1996) 1356.

[15] M. Kawano, H. Ikuno, M. Nishimoto, IEICE Trans. Electron. E76-C (1996) 1358.

[16] C.F. Bohren, D.R. Huffman, Absorption and Scattering of Light by Small Particles, Chap. 8.4, Pergamon Press, 1980.

[17] M. Abramowitz, I.A. Stegun, Handbook of mathematical functions, Chap. 9.1.21, Dover Publications, New York, 1970.

[18] W.C. Chew, Waves and Fields in Inhomogeneous Media, Chap. 1.3.3, IEEE Press, 1994.

[19] E.M. Purcell, C.R. Pennypacker, Astrophys. J. 186 (1973) 705.

[20] G.H. Goedecke, S. O’Brien, Appl. Optics 27 (1988) 2431.

[21] B.T. Draine, P.J. Flatau, J. Opt. Soc. Am. A 11 (1994) 1491.

[22] M.J. Freiser, IEEE Trans. Magn. MAG-4 (1965) 152.

[23] P.S. Pershan, J. Appl. Phys. 38 (1967) 1482.

[24] He. Ping, W.A. McGahan, J.A. Wollam, Optimization of quadrilayer structures for various magneto-optical recording materials, in: Proc. SPIE - The International Society for Optical Engineering, 1991, vol. 1499, pp. 401-411.

[25] N.B. Piller, Optics Lett. 22 (1997) 1674.

[26] A film of the field for the anisotropic example is available on our web site, http:://www.ifh.ee.ethz.ch/ piller/anisotropy $1 . h t m l$. 